\title{
THE THEORY AND EXPERIMENTAL INVESTIGATION OF CONTROLLED SPONTANEOUS CONVERSION NUCLEAR DECAY OF RADIOACTIVE ISOTOPES
}

\author{
V.I. VYSOTSKII \\ Kiev Shevchenko University, Kiev, Ukraine \\ A.A. KORNILOVA, YU. D. PERFILIEV AND L.A. KULIKOV \\ Moscow State University, Moscow, Russia
}

\begin{abstract}
The presented paper discusses the theory and results of direct experimental investigation of the control of internal electron conversion channel of radioactive isotopes spontaneous decay by controlling action of oriented crystal matrix on motion of conversion electrons. The same effect takes place at any $\beta$-processes (including at decay of a neutron with an emission of electrons and neutrino). It was shown that parameters of internal electron conversion decay greatly depend on structure of crystal matrix and on distance from excited nucleus to crystal matrix.
\end{abstract}

\section{Introduction}

It is well known that there are two independent channels of nuclear decay at $E<$ $100 \mathrm{keV}$ : there is a gamma decay channel with a low relative probability $1 /(1+\alpha) \approx$ $10-20 \%$ of decay, and an internal electron conversion channel with a high relative probability $\alpha /(1+\alpha) \approx 90-80 \%$ (where $\alpha \approx 5-10$ is the coefficient of electron conversion for nuclear decay).

Several years ago we created (for the first time) an initial model, ${ }^{1}$ and general theory, ${ }^{2}$ for the influence on the probability of spontaneous gamma decay and the lifetime of gamma-radioactive nuclei in a cavity. We considered the general system, which included: the excited nucleus; the associated electrons; the system of the zero-energy (in vacuum state) electromagnetic modes; and the system of resonant, or non-resonant atoms (screen or cavity), situated at a large distance $L \gg \lambda_{\text {eg }}$ from the excited nucleus. The phenomenon of controlled nucleus gamma decay is a result of the interaction $\mathrm{U}$ of the excited nucleus A with zero-energy electromagnetic modes $F_{i}$, and interaction $V=\sum_{i} V_{i}$ of these modes $F_{i}$ with the atoms of the controlled (and controlling) screen R (see Fig. 1).

It was shown that the decay parameters have a strong dependence on the sign and magnitude of the radiation shift (radiation correction) of the resonance level position (the nuclear analog of the Lamb shift for atomic electrons). It was also shown that the resonant screening effect in all cases appears to be more significant than for the nonresonant one. The greatest influence on the (nucleus) spontaneous 


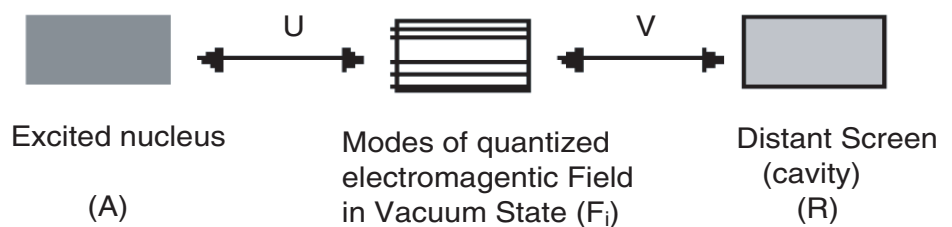

Figure 1. Controlled $\gamma$-decay.

decay process will be realized in the case when the electromagnetic field modes in the zero-energy state, which interact with the nucleus, are mutually synchronized.

In our previous investigations, the phenomenon of controlled spontaneous decay (of the gamma channel) was studied by the indirect methods of intensity, ${ }^{3}$ and spectral width $^{4}$ measurements, of the emitted $\gamma$-radiation; and by the direct method of delayed $\gamma-\gamma$ coincidence $^{5}$. In these experiments we discovered a change (increase) of radiative lifetime of the radioactive nucleus $\mathrm{Fe}^{57 *}$ by $10-40 \%$ (in relation to resonant Mossbauer $\gamma$-channel of decay), and also in the total lifetime (including non-controlled non-Mossbauer $\gamma$-radiation and non-controlled electron conversion channels of decay of the excited nucleus) by $1 \%$. The outcome of the experiment correspond to the predictions of the controlled $\gamma$-decay theory ${ }^{2}$.

This paper discusses the theory of another process (the control of the internal electron conversion channel of spontaneous decay of radioactive isotopes), and results of a direct experimental investigation of this phenomenon. The electron conversion channel plays the main role during the process of spontaneous decay of Mossbauer nuclei. For the first time we have created a theory of the influence on the probability of internal electron conversion decay of radioactive isotopes. The same effect takes place during any beta processes of nuclear decay.

\section{Beta decay of the nucleus in free space}

We considered the general system, which includes the excited nucleus, the system of electrons of this nucleus, and the crystal matrix (Fig. 2). The phenomenon of spontaneous nucleus decay controlling is a result of the interaction $U$ of the excited nucleus A with atomic conversion electrons $E$; and also the interaction $T$ of these emitted fast electrons with the atoms of the controlled (and controlling) crystal matrix $C$ during electron channeling. The probability for the electron conversion channel of nuclear decay (or any other beta decay of nucleus) is ${ }^{1}$

$$
W_{\alpha}=\left(\frac{2 \pi}{\hbar}\right) \sum_{\delta \Omega}\left|T_{\mathrm{ae}}\left(E_{\mathrm{e}}\right)\right|^{2} \rho_{\mathrm{e}}\left(E_{\mathrm{e}}, \delta \Omega\right)
$$




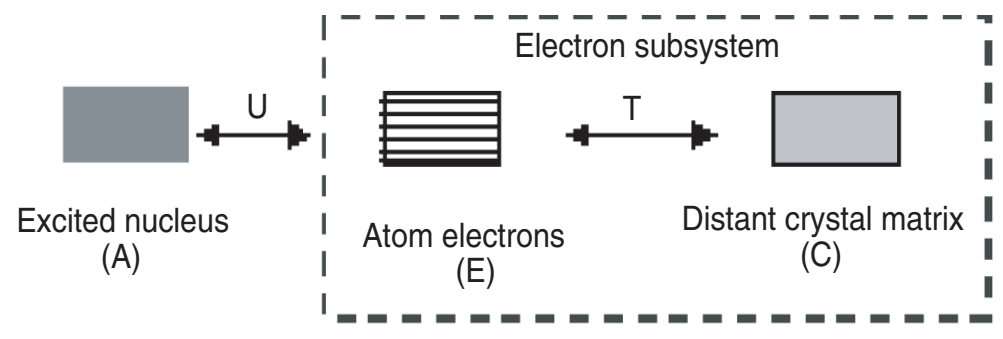

Figure 2. Controlled beta decay.

This rate is proportional to density of final electron states $\rho_{\mathrm{e}}\left(E_{\mathrm{e}} \Omega\right.$ ) (states of free fast electron with energy $E_{\mathrm{e}}$ in direction of solid angle $\Omega$ after decay). In the case of nuclear decay in free space, the density of final electron states on energy and volume is

$$
\rho_{\mathrm{e}(\text { free })}\left(E_{\mathrm{e}}, \delta \Omega\right)=2\left(\int \mathrm{d}^{3} p \int \mathrm{d}^{3} x\right)\left[\frac{\mathrm{V}_{0}}{(2 \pi \hbar)^{3}}\right] \equiv \rho_{\mathrm{e}(\text { free })}\left(E_{\mathrm{e}}\right) \delta \Omega .
$$

Here

$$
\rho_{e(\text { free })}\left(E_{e}\right)=\frac{m p_{\mathrm{e}}}{4 \pi^{3} \hbar^{3}}=\frac{m}{2 \pi^{2} \lambda_{e} \hbar^{2}}
$$

is the density of final electron states in energy, volume and solid angle (number of electron states per single intervals of energy, volume and solid angle),

$$
\lambda_{\mathrm{e}}=\frac{2 \pi \hbar}{p_{\mathrm{e}}}
$$

is the wavelength of emitted free electron that has been taken off from atom (nucleus) during decay, $p_{\mathrm{e}}=E_{\mathrm{e}} / 2 \mathrm{~m}$. In the case of electron conversion channel of nuclear decay the energy of emitted free electron is

$$
E_{\mathrm{e}} \equiv\left(E_{\mathrm{eg}}-e \varphi_{i}\right)
$$

where $E_{\mathrm{eg}}$ is the energy of nuclear transition from excited state to ground state during spontaneous decay, and where $e \varphi_{\mathrm{i}}$ is the initial energy of atom electron in atom before decay. In the case of isotropic decay the probability of beta decay in free space is

$$
W_{\alpha(\text { free })}=\left(\frac{8 \pi^{2}}{\hbar}\right)\left|T_{\mathrm{ae}}\left(E_{\mathrm{e}}\right)\right|^{2} \rho_{\mathrm{e}(\text { free })}\left(E_{\mathrm{e}}\right)
$$




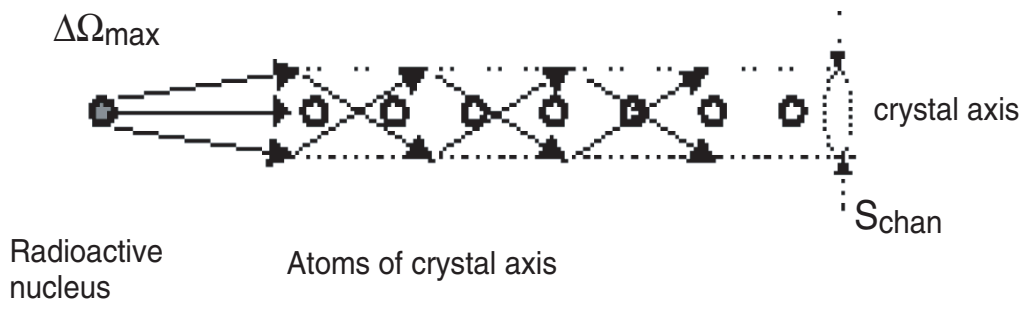

Figure 3. Axial channeling of electron in perfect crystal.

\section{Beta decay of a nucleus in a perfect crystal}

In the presence of a perfect crystal near the excited nucleus, the phenomena of channeling of the created (emitted) free electron takes place (see Fig. 3). The solid angle of channeling is

$$
\Delta \Omega_{\mathrm{ch}} \approx\left(\Delta \theta_{\mathrm{L}}\right)^{2}=\frac{2 \hbar}{p_{\mathrm{e}} d},
$$

where $d$ is interatomic distance in a crystal, where

$$
\Delta \theta_{\mathrm{L}}=\left[\frac{V_{\max }}{\left(p^{2} / 2 m\right)}\right]^{1 / 2}=\left[\frac{2 \hbar}{p_{\mathrm{e}} d}\right]^{1 / 2}
$$

is the Lindhard angle, $V_{\max }$ is the maximal depth of averaged potential well of an axial channel. In the crystal matrix there are many directions for the channeling of emitted free electrons (see Fig. 4).

All emitted nonrelativistic electrons that go into a single channel correspond to one mode of a channeling (i.e. one quantum mechanical state of a motion). If the channeling length equals $L_{z}$, then the spectrum of possible quantized levels of longitudinal electron motion with momentum $p_{\mathrm{ez}}$ is determined by quantized states of longitudinal momentum

$$
p_{\mathrm{ez}}=\frac{2 \pi \hbar}{L_{\mathrm{z}}}
$$

and longitudinal energy

$$
E_{n}=\frac{\left(2 \pi \hbar n / L_{\mathrm{z}}\right)^{2}}{2 m} .
$$

The density of final states of electron subsystem in direction of axial channel is 


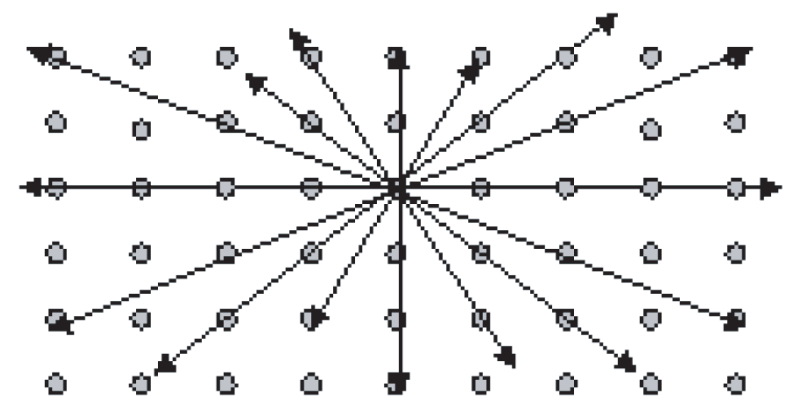

Figure 4. Direction of axial channeling of conversion electrons in a per.

$$
\begin{array}{r}
\rho_{\mathrm{e}_{(\text {chan })}}\left(E_{\mathrm{e}}, \delta \Omega\right)=\frac{\delta \Omega}{\left[\left(S_{\text {chan }} L_{z}\right) \delta E_{\mathrm{n}}\right]}=\left(m / 2 p_{\mathrm{e}} \hbar S_{\text {chan }} \sqrt{\pi}\right) \delta \Omega=\rho_{\mathrm{e}_{(\text {chan })}}\left(E_{\mathrm{e}}\right) \delta \Omega ; \\
\delta \Omega \leqslant \Delta \Omega_{\mathrm{ch}} .
\end{array}
$$

Here

$$
\delta E_{n}=E_{n+1}-E_{n}=2 p \hbar \sqrt{ } \pi / m L_{\mathrm{z}}
$$

is the intermode interval, $S_{\text {chan }}$ is the cross-section of an axial channel and

$$
\rho_{\mathrm{e}(\text { chan })}\left(E_{\mathrm{e}}\right)=\left(m / 2 p_{\mathrm{e}} \hbar S_{\text {chan }} \sqrt{\pi}\right)
$$

is the density of final electron states on energy, volume and solid angle in the direction of axial channel.

The resulting probability of the electron conversion channel of nuclear decay in the presence of a perfect crystal is

$$
W_{\alpha(\mathrm{eff})}=\left(\frac{8 \pi^{2}}{\hbar}\right) \sum_{\delta \Omega}\left|T_{\mathrm{ae}}\left(E_{\mathrm{e}}\right)\right|^{2} \rho_{\mathrm{e}(\mathrm{eff})}\left(E_{\mathrm{e}}\right),
$$

where

$$
\rho_{\mathrm{e}(\mathrm{eff})}\left(E_{\mathrm{e}}\right) \approx\left[1-N\left(\frac{\Delta \Omega_{\mathrm{ch}}}{4 \pi}\right)\right] \rho_{\mathrm{e}\left(E_{\mathrm{e}}\right)}+N\left(\frac{\Delta \Omega_{\mathrm{ch}}}{4 \pi}\right)<\rho_{\mathrm{e}(\operatorname{chan})\left(E_{\mathrm{e}}\right)} .
$$

Here $N$ is the number of directions of electron channeling in perfect crystals that are situated near excited nucleus, 


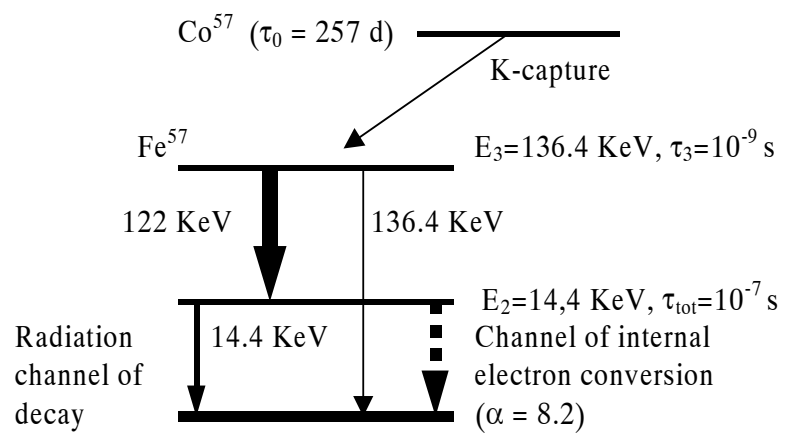

Figure 5. Decay of $\mathrm{Fe}^{57 *}$ isotope.

$$
\left\langle\rho_{\mathrm{e}(\text { chan })}\left(E_{\mathrm{e}}\right)\right\rangle=\left(m / 2 p_{\mathrm{e}} \hbar\left\langle S_{\text {chan }}\right\rangle \sqrt{ } \pi\right)
$$

and $\rho_{\mathrm{e}(\mathrm{eff})}\left(E_{\mathrm{e}}\right)$ are averaged and effective densities of final electron states in energy, volume and solid angle in the direction of axial channeling, $\Delta \Omega_{\mathrm{ch}}=$ $\sum \Delta \Omega, \quad \Delta \Omega_{\text {chan }}$ is a sum of all angles of channeling. For the main crystal axes $<n l k>$ with $n, l, k \leq 3$, we have $N=63$.

The ratio of beta decay in the case of free space and at presence of perfect crystal is

$$
K_{\mathrm{ch}}=\frac{W_{\alpha(\mathrm{eff})}}{W_{\alpha}}=\frac{\rho_{\mathrm{e}(\mathrm{eff})}\left(E_{\mathrm{e}}\right)}{\rho_{\mathrm{e}}\left(E_{\mathrm{e}}\right)}=\left\{1+N \frac{\Delta \Omega_{\mathrm{ch}}}{4 \pi}\left[1-\frac{\left\langle\rho_{\mathrm{e}(\mathrm{chan})}\left(E_{\mathrm{e}}\right)\right\rangle}{\rho_{\mathrm{e}}\left(E_{\mathrm{e}}\right)}\right]\right\} .
$$

From Eq. (7) for $K_{\mathrm{ch}}$ follows that for

$$
\frac{\left\langle\rho_{\mathrm{e}(\text { chan })}\left(E_{\mathrm{e}}\right)\right\rangle}{\rho_{\mathrm{e}}\left(E_{\mathrm{e}}\right)}>1
$$

(or at $\left.\rho_{\mathrm{e}(\mathrm{eff})}\left(E_{\mathrm{e}}\right)>\rho_{\mathrm{e}}\left(E_{\mathrm{e}}\right)\right)$ the presence of a perfect crystal leads to a suppression $\left(K_{\mathrm{ch}}<1\right)$ of the beta decay of the excited nucleus. In the opposite case

$$
\frac{\left\langle\rho_{\mathrm{e}(\text { chan })}\left(E_{\mathrm{e}}\right)\right\rangle}{\rho_{\mathrm{e}}\left(E_{e}\right)}<1
$$

or at $\rho_{\mathrm{e}(\mathrm{eff})}\left(E_{\mathrm{e}}\right)<\rho_{\mathrm{e}}\left(E_{\mathrm{e}}\right)$, the presence of a perfect crystal lead to an acceleration of the decay of the excited nucleus.

\section{Experimental study of controlled beta decay}

The aim of the present experiments was to investigate the controlled internal electron conversion channel of the decay of the radioactive $\mathrm{Fe}^{57 *}$ isotope; with a nuclear transition energy $E_{21} \approx 14.4 \mathrm{keV}$, a coefficient of internal conversion $\alpha \approx 8.2$, energy 

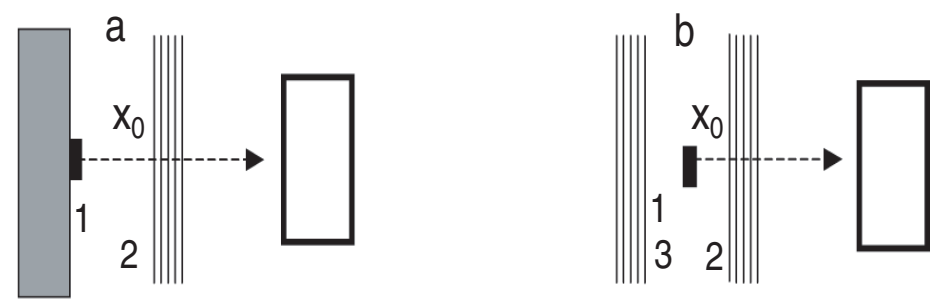

Figure 6. Experimental investigation of controlled beta decay.

$$
E_{\mathrm{e}}=\left(E_{21}-e \varphi_{\mathrm{i}}\right) \approx 6 \mathrm{KeV},
$$

and a wavelength of the conversion electron of approximately $\lambda_{\mathrm{e}} \approx 0.15 \AA$ (Fig. 5).

Investigated isotope source 1 was put on the substrate surface, and covered by very thin (thickness less $50 \mu \mathrm{m}$ ) perfect mica crystal 2 (case $a$ ); or was put in the space between two thin parallel perfect mica crystals 2 and 3 (case $b$ ); with a controlled inter-crystal distance (slot) $x_{0}$ (see Fig. 6). In these experiments we discovered an inhibition of the conversion channel for nuclear decay by $7-10 \%$, and a change (increase) of the total lifetime for the radioactive $\mathrm{Fe}^{57 *}$ isotope by $6-9 \%$, at the optimal size $x_{0}$ of the slot, in relation to spontaneous decay in free space without the thin mica crystal (see Fig. 7). The results of the experiment correspond to the predictions of the theory of controlled spontaneous internal electron conversion decay of Mossbauer nuclei. The same effect takes place in the case of any beta

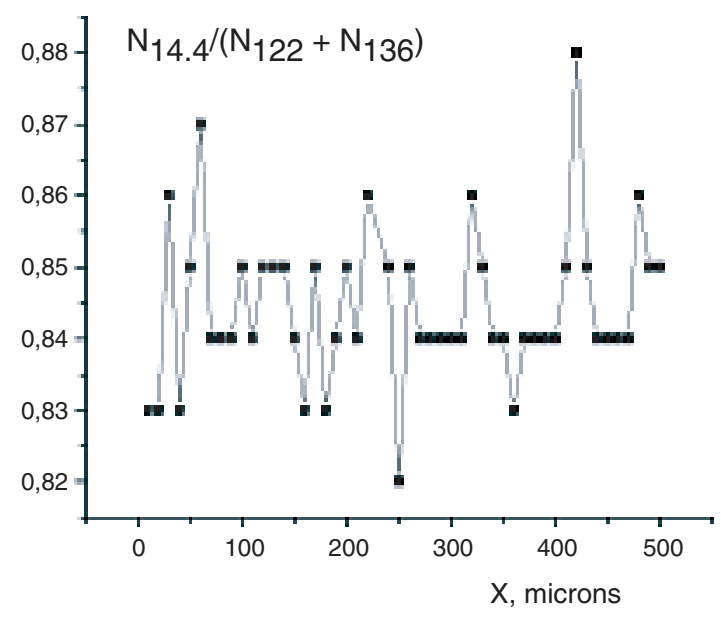

Figure 7 . 
processes (including during the decay of a neutron with the emission of an electron and proton). Using this method it is possible, for example, to increase the free ultracold neutron lifetime in a cavity by making its walls from oriented crystals.

\section{References}

1. V.I. Vysotskii, V.I. Vorontsov and R.N. Kuz'min, Soviet Phys.-J.T.P. Lett. 10, 300 (1984).

2. V.I. Vysotskii, Phys. Rev. 58, 337 (1998).

3. V.I. Vysotskii, V.P. Bugrov, A.A. Kornilova, R.N. Kuz'min and S.I. Reiman, Hyperfine Interactions 107277 (1997).

4. V.I. Vysotskii, V.P. Bugrov, A.A. Kornilova, R.N. Kuz'min and S.I. Reiman, in Proceedings of the International Conference Physics of Nuclear Science and Technology, Vol. 2 (New York, October 5-8, 1998), pp. 1739-1743.

5. V.I. Vysotskii, A.A. Kornilova, A.A. Sorokin, V.A. Komisarova, S.I. Reiman and G.K. Riasnii, Laser Phys. 11(3), 442 (2001). 